Enfield, N. J. (in press). Doing fieldwork on the body, language, and communication.

In C. Müller, E. Fricke, A. Cienki, \& D. McNeill (Eds.),

Handbook Body - Language - Communication.

Berlin: Mouton de Gruyter.

\title{
Doing fieldwork on the body, language, and communication
}

\author{
N. J. Enfield
}

This chapter gives a brief and selective overview of how to approach research on bodily and linguistic aspects of communication by doing fieldwork. Here I do not address technological issues such as specific choices of video recording equipment or computer programs for processing and analysis. Any such discussion would either be too purpose-specific or would quickly become obsolete due to the fast-moving nature of the technology. Our interest here is the general approach.

\section{Available literature on fieldwork}

Extensive and up-to-date resources are available in recent literature on fieldwork in linguistics and language documentation (see, for example, Newman and Ratliff 2001; Crowley 2007; Gippert et al 2007; Vaux et al 2007; Bowern 2008; Dixon 2010; Thieberger 2011; Sakel and Everett 2012). These are all useful for general principles of field research, and to varying extents they show evidence of a current trend in linguistics toward capturing visual aspects of communication. Of special relevance to visual and bodily behavior are Duranti (1997; Chs 4, 5, 8, and 9, and the appendix) and Seyfeddinipur (2012). See also the field manuals developed in the Language and Cognition Group at the Max Planck Institute for Psycholinguistics in Nijmegen (http://fieldmanuals.mpi.nl/); cf. Majid (2012).

\section{Fieldwork defined}

What is fieldwork? One meaning of the term is 'distant travel for research'. This simply refers to the collecting of data somewhere away from the researcher's usual 
place of work, especially when this requires the researcher to travel and be absent from their own home for some time. This is in line with a recent definition given by Majid $(2012,54)$ : 'Fieldwork is the collection of primary data outside of the controlled environments of the laboratory or library'. This notion of fieldwork does not distinguish between qualitatively different modes of data collection. It may, for example, involve a researcher from Germany traveling to a Namibian village to carry out a brief program of controlled experiments on memory for body movements in relation to spatial cognition (Haun and Rapold 2009). Or it may involve a researcher from the Netherlands traveling to central Australia for an extended period of observation and video recording of everyday interaction in Aboriginal communities, to understand how speech and bodily behavior are integrated in referring to space (Wilkins 2003). If this 'travel for research' sense of fieldwork is taken to simply mean that the work is done outside of the research laboratory, it may also refer to work that is done closer to home. We might say, for instance, that we are doing fieldwork when we go downtown to take notes on how people gesticulate in public places (Efron 1941), or when we take a video camera to a local mechanic shop to record workplace interaction in a rich artifactual environment (Streeck 2009; cf. Goodwin 2000).

A second notion of fieldwork is something like 'the recording of researchindependent events'. This refers to the gathering of data by recording events which are taking place independently of the fact that the recordings are being made. This is not to say that the act of recording an event has no impact on the nature of the event. Of course people's behavior can be affected as a result of them knowing that they are being observed. But the idea of fieldwork in this sense is that the events that are being recorded would take place anyway, in roughly the same form, and in the same places and times, even if no research were being conducted on it at all. It might involve recording people cooking, or having dinner, working in their fields or workshops, staging performances or ritual activities. This is distinct from the collection of data using experimental methods (whether one is in the field or not), where the to-berecorded events would not have happened at all were they not instigated by researchers, motivated by research questions and methods.

There are at least two important reasons to do fieldwork in this second sense. First, it can give the researcher access to phenomena that would otherwise be inaccessible, for 
example because they are difficult or impossible to elicit experimentally. Second, and relatedly, this kind of fieldwork provides a way to maximize ecological validity (though, of course, at possible cost to experimental control). The second definition of fieldwork almost entails the first, i.e., that the research be done 'outside the lab' (unless of course one is studying people's communicative behavior in research laboratories). But this is not always the case. On the 'recording research-independent events' definition of fieldwork, we would have to include, for example, the recording of telephone conversations or the gathering of data from web page commentaries.

In this chapter I want to discuss fieldwork in the sense of the overlap between the two notions of the term discussed so far. Thus, we shall focus here on 'travel away from the researcher's home environment to record research-independent events'. Many researchers of bodily behavior have carried out fieldwork in more or less this sense (Kita and Essegbey 2001; Enfield 2003; Haviland 2003; Kendon and Versante 2003; Wilkins 2003, among many others). Seyfeddinipur (2012) provides a linguist-oriented overview and guide of fieldwork on co-speech gesture. There are many useful references there, as well as detailed advice about the recording and annotation of data.

In what follows, I am going to restrict the discussion to this narrower definition than what many people will want to include under 'fieldwork'. In the rest of the chapter I want to discuss some practical points of relevance to carrying out fieldwork on bodily aspects of communication.

\section{Equipment}

For fieldwork on body, language, and communication, by far the most effective method is to use video and sound recording technology. As Hanks (2009) points out, while it is possible to collect data 'on the fly' in the form of handwritten notes, this can only be done if one has already built a sufficient background knowledge of the local cultural and physical setting, and even then the data 'is already a selective interpretation of what the researcher perceives' (Hanks 2009:19). Video recording of interaction gives you the possibility of repeated inspection of the data. Also, with video-recorded data you are able to provide others with direct evidence for your findings and analysis. 
Use the highest quality equipment you possibly can. This will make a huge difference for the quality and longevity of your data. If you invest now in equipment that delivers the best quality possible, it will repay in the long term. Any recording you make today will potentially be a source for your research for many years to come. Especially relevant in research on gesture is your choice of equipment for video recording and digital photography. When selecting equipment, you have to make many choices - what degree of video resolution, what type of lens, which media format, etc.- - and each of these choices represents a potential weak link that may compromise any high-quality choice you have made elsewhere. Even the best sound recording device cannot compensate for the sound delivered through a poor quality microphone. Even the best camera cannot compensate for the poor image delivered through a low quality lens. If the quality of your recordings is compromised in any of these ways, you will be required to live with the limitations of an inferior recording forever.

Quality is priority number one. Beyond this simple rule, it is not possible to give general equipment recommendations. This is partly because your choice of equipment depends on what you are trying to do, and partly because technology is changing so quickly that a good choice today may be inferior or even obsolete tomorrow. In figuring out what equipment to use, first you should specify exactly what your goals are-including what kind of data you want to collect, and what you intend to do with the data afterwards - then study the options, and consult as many colleagues as possible about their experiences. Talk to them and pay attention to what they say. Our more experienced colleagues have paid costs for their lessons learnt, and we can do well to benefit from their costly experiences. Do not repeat others' mistakes.

Even after the best preparation you should expect for things not to go the way you have planned. In fieldwork you must always remain flexible, and be willing to allow your plans to change in an instant. You might be getting ready to make data recordings with a certain goal in mind, yet somehow the circumstances change, and people start doing something different from what they had led you to expect, or they become distracted from what you had hoped they would be doing. In such a case do not get frustrated or try to redirect things. Go with the flow. Like in all empirical 
science, serendipity is a source of new, unexpected insights. In the field, you are in a world that belongs to other people. The dynamics of daily life can be like the changing currents of the surf: If a rip tide takes you, don't fight it. There's no point. It would only weaken you and tire you out. Instead relax and see where the flow leads you. You will soon be able to make your way back to safety.

\section{Ethics of recording human behaviour}

Ethics of fieldwork are complex, with respect to recording people's behaviour, maintaining their privacy, and protecting their identities. There are both legal and moral issues. With regard to the legalities of going into communities and recording people's activities, the fieldworker is subject to specified and binding requirements and restrictions. These are different in different countries. You need to find out what the legalities are, and you must abide by them, otherwise you may be subject to punishment by law, confiscation of data, and damage to your reputation both in your field site and in your professional life. No less important than the legalities are the moral principles at hand. Ethical rules and regulations are there to protect people from harm. Even where you are under no legal constraints, you should always follow the golden rule: don't do things to others that you wouldn't want them to do to you. Imagine yourself being studied in your own home by a foreign guest, and ask yourself how you would want to be treated. Treat others like that. And of course, this rule needs to be calibrated in light of your knowledge of the local cultural setting. The people in your field site may have different sensibilities from you. For instance, while they may not care about being photographed with their shirts off, they might be mortified if you were to publish a picture of them exposing the soles of their feet. Be sensitive.

\section{Getting the data you want}

Before collecting your data, you should be able to state explicitly what you are trying to capture, and why. Do you want everyday conversation? A specific type of religious ritual? Joint activities such as cooking together? Teaching? Are you going to study pointing gestures? Facial expression? Conversational repair? Be explicit about what you are after, and make that your target. There is little point collecting 'general 
purpose' data. Ask yourself: What am I going do with this data? What phenomena am I studying? What publications will I produce based on this data?

When video-recording human behavior in the field, you must ensure that you are getting data of the right kind, and of the best possible quality. These goals are more difficult to meet than one might imagine. Things are easier using the types of fieldwork protocols that are traditional in functional linguistics. These approaches prefer data that are spontaneously produced rather than constructed, and it is normal to elicit such data using non-linguistic stimuli such as pictures, film clips, or concrete objects. With a stimulus-based elicitation protocol, the researcher can bring the participant to a relatively quiet place, and can fairly easily control the production of communicative behavior - the when, where, what, who, and how (see for instance, the approaches described in Payne 1997; Majid 2012). Nine out of ten times, such a recording will be successful. But when you want to record unelicited, unscripted, independently occurring behavior in everyday life, it is the opposite. So many things can go wrong.

I recommend following a ' $1 / 10$ Rule'. For every ten recordings you make, expect that one of them will work out well, while in nine of them something will go wrong. Expect one out of ten recordings to be good enough. Of course, with practice one can improve the odds, but if you expect a low success rate you can ensure that the quality stays high, and if you happen to do better, you will be pleasantly surprised.

Traditional linguistic fieldwork is like farming. One cultivates one's data. By making appointments with consultants, and determining a line of questioning, you are taking a direct hand in managing the acquisition of data. But if you are going into the wild, you do not cultivate the things you want, instead you arrange to be where and when they can be captured. There are two ways to go about this: hunting, and trapping. If you try and chase the action like a hunter you will usually be frustrated. Instead, take the approach of a trapper. Study the routines of your quarry. Learn when and where your target type of data tends to recur, and then set up your equipment at those places and times. For example, you might observe that between 4 and 5 o'clock most afternoons, during a certain time of year, a certain group of men will sit and chat on the verandah of a certain house after they have arrived back in the village from their 
day's work in the fields. You can set up your camera here before they arrive. If you are chasing the data instead of lying in wait, you will often miss where a sequence begins, as you'll only recognize it once it's already started. But if your equipment is already set up where human interaction is most likely to occur, then you maximize your chances of capturing an interaction from its inception. Like a trapper, be patient. Let the camera roll even when nothing special is happening, and walk away. Come back later and find out what you have captured.

Be prepared to catch something other than what you were after, or maybe even to catch nothing at all. In one case, I recall, I set up a camera in a village household, and soon after I left the scene, so did all the people who I had hoped to film. The result was an hour of footage of an empty room. But the costs of these kinds of failures are negligible: a bit of time is lost, and a videocassette or small section of hard drive is filled up. With digital media, now the norm, your only constraint is hard-disk space. You can easily ensure that you have ample space for many more recordings than you will actually end up using.

\section{Basic approach to video recording}

Quality should be your highest priority. Collecting recordings is an investment, and if the quality is good, you will long be grateful for your own efforts. Conversely, if the quality is bad you will be frustrated by your data forever. Remember that you are collecting data for scientific research. Do not confuse your data recordings with the kind of informal documentary recordings of your field site in which you might be guided by more aesthetic considerations. By all means collect general recordings of everyday life in the village - indeed community members typically love to see these; or perhaps you want to make a documentary film about your field site. But keep this quite separate from your data collection, where the priority is getting the clearest possible image of the data you are aiming to collect. If you are busy trying to get a close-up of a speaker's face for posterity you will later curse these efforts when you are unable to see what the speaker was doing with his hands.

One fundamental problem to avoid is bad lighting. Often a recording is too dark. This can happen if the recording is made in a room with small windows and no lights. It 
often happens that the lighting of a recording is poor because the camera is pointing toward the main source of light. You can avoid this 'back-lighting' problem by setting up the camera with the main light source behind it. From example if you are filming inside a room where daylight is coming in through a window, set up the camera at the window, pointing into the room, so that the light is coming in from behind the camera. Never set up the camera to be pointing toward the window, unless you are very sure you have made the right kind of technical adjustments for back-lighting on the camera. The same principle of back-lighting applies in many other situations, e.g., when filming outside under a tree or verandah. The rule is, as far as possible, try to have the main source of light, whether it be daylight or artificial light, coming from behind the camera.

Another problem is camera movement. Even small movements of the camera will create problems for your analysis, blurring the image. Try to avoid moving the camera by panning, tilting, or zooming in and out. Make sure that all the action is included in the frame. Leave just enough space free where people might put their hands if, for example, they were to make a large pointing gesture. Don't cut people's body parts from the frame, at least where these would be relevant to communication and normally visible to people in the situation. Avoid hand-held recordings if possible, i.e., by using a tripod. However, sometimes making hand-held recording has advantages, in which case you should be careful to brace the camera and avoid camera shake as far as possible.

Some final points concern the preparation for, and management of, your recordings. When in the field, make sure you have your recording equipment fully ready at all times. This means that whenever you have time, for example before you go to bed at night, make all the necessary preparations in advance for your next set of recordings: for example, fully charge all the necessary devices and batteries, pack your work bag with all the things you need, such as extra blank videotapes, or formatted memory cards, depending on the kind of equipment you are using. Your work bag is then ready for you to grab at any moment. Check and re-check that everything is in working order. And when you are actually making your recordings, you should not only check and re-check, but re-re-check as well. Are your batteries charged? Are your lighting and focus settings correct? Is your framing good? And particularly 
important is the input of sound to your video recorder. If you use an external microphone, you can have a better quality microphone than the one in your camera, and you will be able to place it closer to the action. However, it is easy to make errors with cable connections and sound settings, and so it is crucial to check, re-check, and re-re-check that your sound input during recording is working well. Use headphones to monitor the sound input on the camera once you have begun any recording.

Whenever you make a recording, you should immediately note down the relevant metadata: the time and place of the recording, what are the activities being recorded, who the people are, and any other possibly relevant information. You can easily and quickly note these things at the time of recording, and if you don't, you will find it difficult if not impossible to remember all the relevant details later. Lastly, backup your data as soon as you can. And keep your backups in a different place from your original data, especially when you are traveling.

\section{Closing remark}

This chapter has offered some remarks concerning fieldwork on the body, language, and communication. There are many significant and complex issues that I have not mentioned. If you are intending to do fieldwork, it is worth reading widely beforehand, and drawing on others' experiences as far as you can. But nothing is as valuable as first-hand experience. If you want to do fieldwork, just do it. No matter how well-prepared you are, you will make mistakes. Just make sure you learn from these mistakes. Don't try to do everything in a single field trip. Take time in between, in order to assess your experience and adjust your way of working. And above all, remain flexible and good-humored. Rigidity and stress are both unhealthy and contagious: they should be avoided at all costs.

\section{Acknowledgements}

Thank you to Nick Williams for helpful comments on an earlier draft, and to colleagues in the Language and Cognition Department at the Max Planck Institute for Psycholinguistics in Nijmegen for their input during many discussions on the topics 
discussed here. And thanks to Julija Baranova for expert assistance. This work is supported by the European Research Council (Grant 'Human Sociality and Systems of Language Use').

\section{References}

Bowern, Claire. 2008. Linguistic Fieldwork: A Practical Guide. Palgrave Macmillan. Crowley, Terry. 2007. Field Linguistics: A Beginner's Guide. Oxford: Oxford University Press.

Dixon, R. M. W. 2010. Basic Linguistic Theory. Oxford: Oxford University Press.

Duranti, Allesandro. 1997. Linguistic anthropology. Cambridge: Cambridge University Press.

Efron, David. 1941. Gesture, Race, and Culture: a tentative study of some of the spatio-temporal and "linguistic" aspects of the gestural behavior of Eastern Jews and Southern Italians in New York City, living under similar as well as different environmental conditions. The Hague/Paris: Mouton.

Enfield, N. J. 2003. "Demonstratives in space and interaction: data from Lao speakers and implications for semantic analysis." Language 79 (1): 82-117.

Gippert, Jost, Nikolaus P. Himmelmann, Ulrike Mosel (eds). 2006. Essentials of Language Documentation. Berlin and New York: De Gruyter.

Goodwin, Charles. 2000. Action and embodiment within situated human interaction. Journal of Pragmatics 32: 1489-1522.

Hanks, William F. 2009. Fieldwork on deixis. Journal of Pragmatics 41: 10-24.

Haun, Daniel B. M., and Christian J. Rapold. 2009. "Variation in memory for body movements across cultures." Current Biology 19 (23): R1068-R1069. doi:10.1016/j.cub.2009.10.041.

Haviland, John. 2003. How to point in Zinacantan. In Pointing: where language, culture, and cognition meet, ed. Sotaro Kita. Mahwah: Lawrence Erlbaum.

Kendon, Adam, and Laura Versante. 2003. Pointing by hand in "Neapolitan." In Pointing, ed. Sotaro Kita, 109-138. Mahwah, NJ: Lawrence Erlbaum.

Kita, Sotaro, and James Essegbey. 2001. "Pointing left in Ghana: How a taboo on the use of the left hand influences gestural practice." Gesture 1 (1): 73-94. 
Majid, Asifa. 2012. A guide to stimulus-based elicitation for semantic categories. In The Oxford Handbook of Linguistic Fieldwork, 54-71. Oxford: Oxford University Press.

Newman, Paul and Martha Ratliff (eds). 2001. Linguistic Fieldwork. Cambridge: Cambridge University Press.

Payne, Thomas E. 1997. Describing morphosyntax: a guide for field linguists. Cambridge: Cambridge University Press.

Sakel, Jeanette, and Daniel L. Everett. 2012. Linguistic Fieldwork: A Student Guide. Cambridge: Cambridge University Press.

Seyfeddinipur, Mandana. 2012. Reasons for documenting gestures and suggestions for how to go about it. In The Oxford Handbook of Linguistic Fieldwork, 147165. Oxford: Oxford University Press.

Streeck, Jürgen. 2009. Gesturecraft: the manu-facture of meaning. Amsterdam and Philadelphia: John Benjamins.

Thieberger, Nicholas, ed. 2011. The Oxford Handbook of Linguistic Fieldwork. Oxford: Oxford University Press.

Vaux, Bert, Justin Cooper and Emily Tucker (eds). 2007. Linguistic Field Methods. Eugene: Wipf and Stock.

Wilkins, David P. 2003. Why pointing with the index finger is not a universal (in socio-cultural and semiotic terms). In Pointing, ed. Sotaro Kita, 171-216. Mahwah, NJ: Lawrence Erlbaum Associates. 